\title{
An MHD stagnation slip flow on a moving plate.
}

\begin{abstract}
In this paper, the problem of a steady laminar boundary layer flow of a an electrically conducting fluid in the presence of a magnetic field near the stagnation point with slip on a moving plate is studied. The transformed boundary layer equations are solved numerically using the shooting method. Numerical results are obtained for various values of the magnetic parameter $M$ and the slip factor $\lambda$. The skin friction coefficients and the velocity profiles $f^{\prime}(\eta)$, $\mathrm{g}(\eta)$ and $\mathrm{h}(\eta)$ for various values of $\mathrm{M}$ and $\lambda$ are obtained and discussed.
\end{abstract}

Keyword: MHD; Boundary layer; Stagnation point. 